\title{
Tube feeding outcomes of infants in a Level IV NICU
}

\author{
Sadie L. Williams ${ }^{1,2,3} \cdot$ Natalie M. Popowics ${ }^{4}$ Dawit G. Tadesse ${ }^{5} \cdot$ Brenda B. Poindexter $^{1,2} \cdot$ Stephanie L. Merhar ${ }^{1,2}$
}

Received: 22 April 2019 / Revised: 4 June 2019 / Accepted: 12 June 2019 / Published online: 6 August 2019

(c) The Author(s), under exclusive licence to Springer Nature America, Inc. 2019

Abstract

Background NICU patients are commonly discharged home with nasogastric (NG) or gastrostomy (G-tube) feeding, but wide practice variation exists. The objective of this study was to evaluate feeding and growth outcomes and complications in NICU patients discharged home with NG or G-tube feeding.

Study design Retrospective cohort study of infants discharged from a Level IV NICU with an NG or G-tube who had follow up to 1 year. Clinical characteristics and outcomes were compared between groups.

Results The study sample included 264 infants: 140 with NG and 124 with G-tube. More infants in the G-tube group (65\%) still required tube feedings 12 months post-discharge than infants in the NG group (24\%). Infants in the G-tube group had more tube-related ER visits than infants in the NG group. Growth outcomes did not differ.

Conclusion Home NG feeding may be a safe alternative to a surgically placed G-tube in select NICU patients.

\section{Introduction}

Oral feeding is a multifaceted skill that may take days to months for infants hospitalized in the neonatal intensive care unit (NICU) to master. Complex neural pathways and multiple muscles are required for the coordination of aerodigestive functions [1]. Many of these pathways are immature in the premature neonate and may not be optimally functioning in infants with other congenital or acquired disorders $[1,2]$. Infants who are able to tolerate enteral nutrition but are unable to take the full volume orally are commonly fed via orogastric (OG) or nasogastric (NG) tubes while hospitalized. Infants who are unable to achieve full volume feedings by mouth after adequate time for maturation and acquisition of skills may undergo surgical placement of a gastrostomy tube (G-tube) prior to hospital

Stephanie L. Merhar

stephanie.merhar@cchmc.org

1 Perinatal Institute, Division of Neonatology, Cincinnati Children's Hospital Medical Center, Cincinnati, OH, USA

2 University of Cincinnati Department of Pediatrics, Cincinnati, $\mathrm{OH}$, USA

3 Advent Health for Children, Orlando, FL, USA

4 Duke University, Durham, NC, USA

5 Division of Epidemiology and Biostatistics, Cincinnati Children's Hospital Medical Center, Cincinnati, OH, USA discharge. However, G-tube placement is a surgical procedure with risks of infection, leaking, poor wound healing, and anesthetic complications [3, 4]. Likewise, home NG use is not without risks. Accidental NG removal is common and repeated insertion may lead to trauma or misplacement $[5,6]$ and long term use of NG tubes may lead to oral aversion [7].

Although there is wide practice variation with home NG feedings [8], few studies exist related to the safety and effectiveness of home NG feeding [9-11]. The decision to send a NICU patient home with an NG tube or proceed with G-tube placement affects length of stay [12], cost of care [13], and the family's daily life after discharge. The purpose of this study was to evaluate feeding and growth outcomes and assess potential complications in patients discharged home with NG or G-tube feedings from a large quarternary NICU.

\section{Patients and methods}

\section{Study population}

All infants discharged between 1 January 2016 and 30 June 2017 from the Cincinnati Children's Hospital Medical Center (CCHMC) Level IV NICU with an NG or G-tube were identified. Most infants in both groups were orally feeding in addition to these tube feeds. Infants were included in the analysis for this retrospective chart review if they 
were followed in a CCHMC outpatient clinic for at least two visits in the year after discharge. Infants who died prior to initial hospital discharge, were sent home on hospice, went home with TPN or a GJ tube, were not followed in a CCHMC follow up clinic or had a tracheoesophageal fistula with G-tube placed before 7 days of life were excluded from the study.

Infants were sent home on NG feedings per the discretion of the attending physician, but a guideline was in place with the following parameters: safe to orally feed (PO) as determined by the speech therapists and attending physician, taking $\geq 50 \%$ by mouth and demonstrating continued progress with their oral intake, family had the adequate resources and education to provide NG feedings at home, follow-up arranged with the NICU Follow up Clinic or a primary care provider comfortable with managing feedings, and attending physician discussed the criteria for G-tube placement post-discharge with the family if the infant did not reach full PO feedings at home. G-tube placement was generally recommended by the NICU Follow up Clinic provider as an outpatient if the infant did not make significant progress or regressed in oral feeding ability after 1-2 months of home NG feedings.

The primary outcome was the percentage of infants still requiring tube feedings at 12 months corrected age. There were several secondary outcomes evaluated. We evaluated the number of feeding tube-related emergency room (ER) visits and admission in the first year post initial hospital discharge. CCHMC is the only pediatric hospital in the region so the majority of ER visits and all admissions were at CCHMC. Visits to other local ERs were visible in the CCHMC patient record through CareEverywhere. We defined "tube-related" as visits or admissions where the chief complaint was tube dislodgement, tube leakage, infection at tube site, skin issues related to tube (e.g., granulation tissue or bleeding around $\mathrm{G}$ tube site), or significant feeding intolerance. We also included visits/ admissions for enterocutaneous fistula leakage or closure after G tube removal as "feeding tube-related", if within 12 months of initial hospital discharge. We evaluated growth at 12 months (+I- 1 month) corrected age (Z-score weight, length and head circumference based on the WHO growth curves).

Additional information collected from the chart included sex, gestational age at birth, birth weight, congenital anomalies, and comorbidities, day of life of commencement of enteral nutrition and oral feedings, number of days on mechanical ventilation, chronic lung disease defined as respiratory support at 36 weeks corrected age, surgeries while in the NICU, corrected age at G-tube placement (if G-tube placed), weight, length and head circumference and Z-scores based on the WHO growth curve at 44 weeks corrected age and hospital discharge, type of feeding and fortification at discharge, the use of anti-reflux medication at discharge, respiratory support at discharge. Other post-discharge information collected, if relevant, included date full oral feedings were achieved, date of G-tube removal, and post-discharge feeding therapies.

The CCHMC Institutional Review Board reviewed this retrospective study and approved it with a waiver of informed consent. Chart review was performed and data was entered into a secure electronic (Research Electronic Data Capture, REDCap) database created specifically for this project.

\section{Statistical analysis}

We compared infants discharged with NG with those discharged with G-tube feedings. We conducted statistical analysis in StataSE 15 and RStudio. We assessed significance at the 0.05 level and all tests were two-tailed. We used $t$-tests for continuous variables and Fisher's exact test for discrete variables. We performed survival analysis for the outcome of "days after discharge to full oral feedings" using the Kaplan-Meier method. We performed backward stepwise logistic regression to determine predictors of reaching full oral feedings by 2 months post hospital discharge.

\section{Results}

A total of 326 infants were discharged from the CCHMC NICU with either an NG or G-tube between January 2016 and 30 June 2017. Of these infants, 62 were excluded from the study (see Fig. 1 for a flow chart of the study population). The remaining 264 infants were included in the analysis: 140 infants discharged with an NG tube and 124 infants were discharged with a G-tube. Patient characteristics are listed in Table 1.

\section{Feeding outcomes}

The percentage of infants still requiring tube feedings at 12 months post hospital discharge was higher in those discharged with G-tubes $(65 \%)$ compared with NG tubes (24\%), $p<0.001$. Twenty-nine infants in the NG group (21\%) had a $\mathrm{G}$ tube placed at some point in the 12 months after initial hospital discharge. We performed a survival analysis to evaluate how long it took infants in each of the three groups (home with NG no G tube, home with NG later $\mathrm{G}$ tube, home with $\mathrm{G}$ tube) to attain full oral feeds (Fig. 2). As shown in the figure, the majority $(>60 \%)$ of infants discharged with NG feeds attained full oral feeds within 60 days of hospital discharge. 


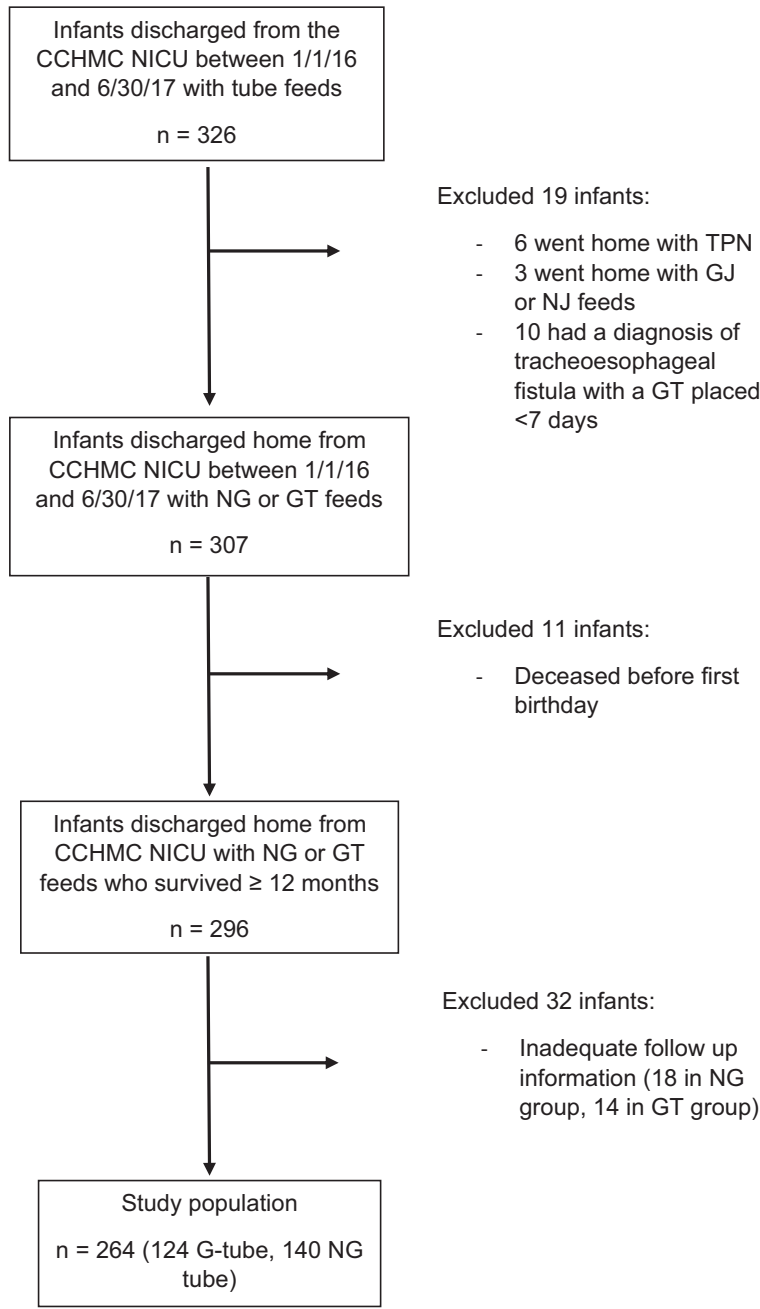

Fig. 1 Flow chart of study population

We then divided the infants into two groups, regardless of whether they went home with an NG or G tube: those who reached full oral feeds within 60 days of hospital discharge $(n=99)$ and those who did not $(n=165)$. We performed a backward stepwise logistic regression to evaluate factors that contributed to attainment of full oral feeds by 60 days. Covariates considered for the regression model included: sex, gestational age at birth, neurologic comorbidities, cardiac comorbidities, congenital diaphragmatic hernia, chronic lung disease, gastrointestinal comorbidities, Pierre-Robin syndrome, renal comorbidities, Trisomy 21 or 18 , percent oral feeds on the day prior to discharge, respiratory support at discharge, receiving any maternal breast milk at discharge, and receiving anti-reflux medications at discharge. The final model showed that higher percentage of oral feeds at discharge, Pierre Robin syndrome, and receiving maternal breast milk at discharge were positively associated with attaining full oral feeds by 60 days, while chronic lung disease, more respiratory support at
Table 1 Patient characteristics

\begin{tabular}{|c|c|c|c|}
\hline Variable & $\begin{array}{l}\text { NG tube } \\
(n=140)\end{array}$ & $\begin{array}{l}\mathrm{G} \text { tube } \\
(n=124)\end{array}$ & $p$-value \\
\hline Sex (male) & $76(54 \%)$ & $72(58 \%)$ & 0.62 \\
\hline $\begin{array}{l}\text { Gestational age at birth } \\
\text { (weeks) }\end{array}$ & $35.7(4.1)$ & $33.1(5.6)$ & $<0.001$ \\
\hline $\begin{array}{l}\text { Corrected age at discharge } \\
\text { (weeks) }\end{array}$ & $5.5(5.2)$ & $15.5(14.4)$ & $<0.001$ \\
\hline Ventilator days & $18.2(86.9)$ & $188.2(354.3)$ & $<0.001$ \\
\hline $\begin{array}{l}\% \text { oral feeds on the day } \\
\text { prior to discharge }\end{array}$ & $42.8(26.7)$ & $14.4(24.4)$ & $<0.001$ \\
\hline $\begin{array}{l}\text { Reflux medications at } \\
\text { discharge }\end{array}$ & $45(32 \%)$ & $55(44 \%)$ & 0.04 \\
\hline $\begin{array}{l}\text { Respiratory support at } \\
\text { discharge }\end{array}$ & & & $<\mathbf{0 . 0 0 1}^{\text {b }}$ \\
\hline None & $111(79 \%)$ & $60(48 \%)$ & \\
\hline Nasal cannula & $27(19 \%)$ & $27(22 \%)$ & \\
\hline $\begin{array}{l}\text { Tracheostomy }+/- \\
\text { ventilator }\end{array}$ & $2(1 \%)$ & $37(30 \%)$ & \\
\hline \multicolumn{4}{|l|}{$\begin{array}{l}\text { Congenital anomalies/ } \\
\text { comorbidities }\end{array}$} \\
\hline Neurologic & $40(29 \%)$ & $31(22 \%)$ & 0.58 \\
\hline Cardiac & $21(15 \%)$ & $16(13 \%)$ & 0.72 \\
\hline Chronic lung disease & $13(9 \%)$ & $42(34 \%)$ & $<0.001$ \\
\hline $\begin{array}{l}\text { Congenital } \\
\text { diaphragmatic hernia }\end{array}$ & $16(11 \%)$ & $5(4 \%)$ & 0.04 \\
\hline Gastrointestinal & $14(10 \%)$ & $16(13 \%)$ & 0.56 \\
\hline Craniofacial & $11(8 \%)$ & $10(8 \%)$ & 1.0 \\
\hline Renal & $6(4 \%)$ & $6(5 \%)$ & 1.0 \\
\hline Genetic & $44(31 \%)$ & $50(40 \%)$ & 0.16 \\
\hline Prematurity < 37 weeks & $68(49 \%)$ & $74(60 \%)$ & 0.08 \\
\hline
\end{tabular}

Bold values indicate statistical significance $p<0.05$

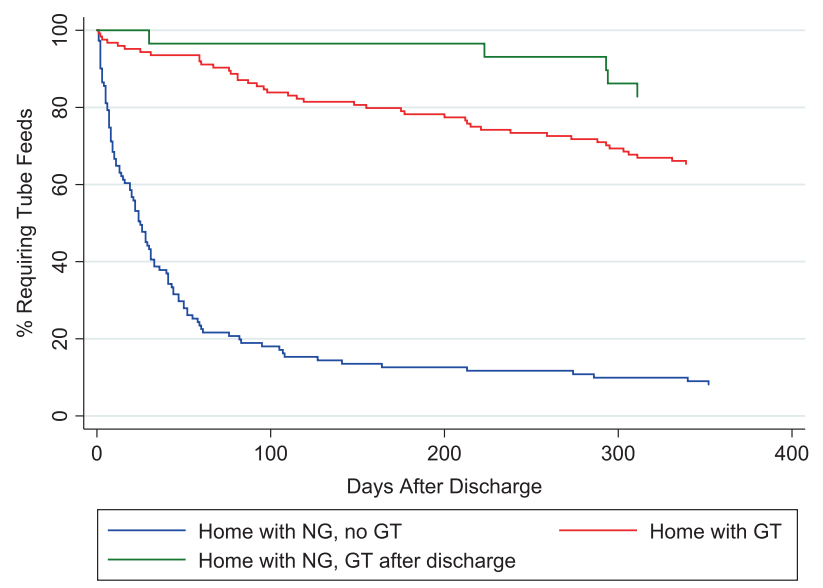

Fig. 2 Kaplan-Meier curve showing days to full oral feeds

discharge, and receiving anti-reflux medications at discharge were negatively associated with attaining full oral feeds by 60 days. 


\section{Growth outcomes}

As shown in Fig. 3, growth outcomes did not differ between the two groups at any of the three time points (44 weeks corrected age, hospital discharge, or 12 months corrected age).

\section{ER visits and readmissions related to feeding}

As shown in Table 2, infants discharged with $G$ tubes had significantly more ER visits related to the feeding tube (tube dislodgment, tube malfunction, skin irritation, and infection) in the 12 months after initial hospital discharge than infants discharged with NG tubes. Five infants in the G tube group had five ER visits each related to the tube in the first year after discharge. There was a trend toward more admissions related to feeding (tube issues as above or feeding intolerance) in the first year after discharge in the $\mathrm{G}$ tube group, but this was not significant.

\section{Discussion}

In this retrospective cohort study, we evaluated all infants discharged from a single Level IV NICU over an 18 month period with a G-tube or NG tube in place. We found that significantly more infants discharged home with G-tubes still required tube feeds at 12 months after discharge compared to those discharged with NG tubes. This finding was expected, as infants discharged home with G-tubes were generally sicker, more premature, and had more comorbidities.

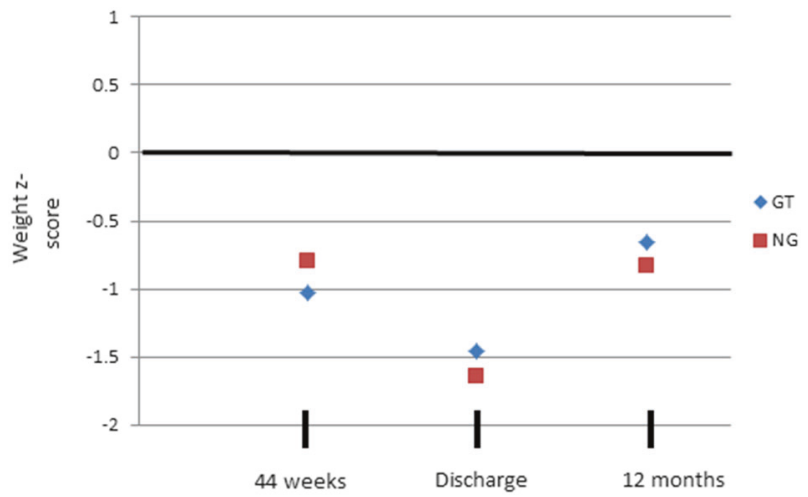

Fig. 3 Growth outcomes by type of feeding tube
However, a surprising percentage $(9 \%)$ of infants discharged with $G$ tubes attained full oral feeds within 60 days after discharge. Given the potential complications associated with surgery in infants, this suggests that some G-tube placements can be avoided, instead relying on home NG feeding as an alternative [10, 11]. Using logistic regression, we evaluated factors associated with achieving full oral feeds within 60 days of hospital discharge in infants discharged home with either type of tube, and found that the percentage of oral feeds, receiving maternal breast milk at discharge, and the diagnosis of Pierre-Robin syndrome were predictive of reaching full oral feeds quickly, while the amount of respiratory support, a diagnosis of chronic lung disease, and receiving anti-reflux medications at discharge were predictive of still requiring tube feeds at 60 days after discharge. This suggests that home NG feeds may be a particularly good alternative in infants discharged home on room air or nasal cannula who are taking some percentage of feeds orally. The association with maternal breast milk likely reflects the fact that those infants discharged still receiving breast milk had shorter hospital stays and therefore less severe illness. Conversely, those infants discharged home on anti-reflux medications likely had more prolonged hospital stays, and likely more symptoms of reflux, which can lead to oral aversion, accounting for the association. We treat Pierre-Robin syndrome with either internal or external mandibular jaw distraction in our NICU, and infants usually take some time to regain oral skills after distractor removal but are often successful at reaching full oral feeds, reinforcing our practice of discharging most infants with Pierre-Robin with an NG rather than placing a $\mathrm{G}$ tube in the NICU. The clinical guideline in our NICU recommends that infants should be taking over $50 \%$ of feeds orally in order to go home with NG feeds, but our data show that the average percentage of oral feeds for the NG group was only $42.7 \%$, suggesting that clinicians often send infants home with NG feedings who are taking less than $50 \%$ by mouth. Anecdotally we find that infants do better with oral feeds at home when they have a consistent person feeding them each time (versus varying nurses or therapists while in the NICU), so it is not surprising that many infants were able to reach full oral feeds quickly at home. However, the prolonged presence of an NG tube may increase the development of feeding aversion [7]. Our data suggest that if infants sent home with NG tubes do not reach full oral

Table 2 ER visits related to feeding tube in 12 months after hospital discharge

\begin{tabular}{|c|c|c|c|}
\hline Variable & NG tube $(n=140)$ & G tube $(n=124)$ & $p$-value \\
\hline \# of infants with ER visits related to feeding tube in first 12 months after discharge & 15 & 71 & $<0.001$ \\
\hline \# of infants with $>1$ ER visit related to feeding tube in first 12 months after hospital discharge & 5 & 29 & $<0.001$ \\
\hline Hospital readmissions related to feeding in first 12 months after hospital discharge & 9 & 16 & 0.09 \\
\hline
\end{tabular}

Bold values indicate statistical significance $p<0.05$ 
feedings by 60 days after hospital discharge, they are likely to require tube feeds for an extended period and would benefit from a surgically placed G-tube.

Infants discharged home with an NG had significantly fewer tube-related ER visits in the first 12 months compared to those infants discharged with a G-tube. Our hospital has developed educational materials on G-tubes for families and processes to decrease ER visits related to G-tubes, but our data show that in this cohort, over half of infants sent home with G-tubes had a tube-related ER visit within the first 12 months after discharge and there were five infants in the G-tube group who had five ER visits related to the tube, representing a significant cost and use of medical resources. This data suggests a need for better education for families on G-tube care and more resources available to families to help avoid costly ER visits.

Overall, growth was not significantly different between the groups at all three time points studied: 44 weeks corrected age, hospital discharge, and 12 months corrected age. This is reassuring in that infants being sent home with NG feedings, despite reaching full oral feeds earlier, are able to maintain an appropriate growth trajectory.

Strengths of this study include a large sample size and extensive follow-up information because our center is the only children's hospital in our metropolitan area. Limitations of the study include its retrospective nature and data collected from a single center, limiting generalizability. In addition, children discharged with NG and G-tubes from our hospital certainly represent different populations, making some types of comparisons difficult.

As not all infants with home NG feeds were able to achieve full oral feeds and not all infants discharged with Gtubes required prolonged tube feeding, this study emphasizes the need for further studies to evaluate the factors related to the need for continued tube feeding, which may help clinicians decide when to send infants home with NG feeds versus recommending G-tube placement before hospital discharge. This work also highlights potential areas for quality improvement projects related to reducing the numbers of ER visits in children sent home with G tubes.

\section{Conclusion}

Home NG feeding may be a safe and cost-effective alternative to a surgically placed G-tube in select NICU patients.

\section{Compliance with ethical standards}

Conflict of interest The authors declare that they have no conflict of interest.

Publisher's note: Springer Nature remains neutral with regard to jurisdictional claims in published maps and institutional affiliations.

\section{References}

1. Jadcherla S. Dysphagia in the high-risk infant: potential factors and mechanisms. Am J Clin Nutr. 2016;103:622S-8S.

2. da Costa SP, van den Engel-Hoek L, Bos AF. Sucking and swallowing in infants and diagnostic tools. J Perinatol. 2008;28:247-57.

3. Correa JA, Fallon SC, Murphy KM, Victorian VA, Bisset GS, Vasudevan SA, et al. Resource utilization after gastrostomy tube placement: defining areas of improvement for future quality improvement projects. J Pedia Surg. 2014;49:1598-601.

4. Crosby J, Duerksen D. A retrospective survey of tube-related complications in patients receiving long-term home enteral nutrition. Dig Dis Sci. 2005;50:1712-7.

5. Quandt D, Schraner T, Ulrich Bucher H, Arlettaz Mieth R. Malposition of feeding tubes in neonates: is it an issue? J Pedia Gastroenterol Nutr. 2009;48:608-11.

6. Parker LA, Withers JH, Talaga E. Comparison of neonatal nursing practices for determining feeding tube insertion length and verifying gastric placement with current best evidence. Adv Neonatal Care. 2018;18:307-17.

7. Hoogewerf M, Ter Horst HJ, Groen H, Nieuwenhuis T, Bos AF, van Dijk MWG. The prevalence of feeding problems in children formerly treated in a neonatal intensive care unit. J Perinatol 2017;37:578-84.

8. Greene NH, Greenberg RG, O'Brien SM, Kemper AR, Miranda ML, Clark RH, et al. Variation in gastrostomy tube placement in premature infants in the United States. Am J Perinatol. 2018. [Epub ahead of print]

9. González-Gil T. Early discharge with home support of gavage feeding for stable preterm infants who have not established full oral feeds. Int J Evid Based Healthc. 2016;14:34-5.

10. Rosen D, Schneider R, Bao R, Burke P, Ceballos C, Hoffstadter-Thal K, et al. Home nasogastric feeds: feeding status and growth outcomes in a pediatric population. J Parent Enter Nutr. 2016;40:350-4.

11. Khalil ST, Uhing MR, Duesing L, Visotcky A, Tarima S, Nghiem-Rao TH. Outcomes of infants with home tube feeding: comparing nasogastric vs gastrostomy tubes. J Parent Enter Nutr. 2017;41:1380-5.

12. Fox D, Campagna EJ, Friedlander J, Partrick DA, Rees DI, Kempe A. National trends and outcomes of pediatric gastrostomy tube placement. J Pedia Gastroenterol Nutr. 2014;59:582-8.

13. Landisch RM, Colwell RC, Densmore JC. Infant gastrostomy outcomes: the cost of complications. J Pedia Surg. 2016; 51:1976-82. 